\title{
Predicting responses to sunitinib using single nucleotide polymorphisms: progress and recommendations for future trials
}

\author{
Ram N Ganapathi* and Ronald M Bukowski
}

\begin{abstract}
Targeted therapy with tyrosine kinase inhibitors has led to a substantial improvement in the standard of care for patients with advanced or metastatic clear cell renal cell carcinoma. Because the mechanism of action, metabolism and transport of tyrosine kinase inhibitors can affect outcome and toxicity, several investigators have pursued the identification of single nucleotide polymorphisms (SNPS) in genes associated with these actions. We discuss SNPs associated with outcome and toxicity following sunitinib therapy and provide recommendations for future trials to facilitate the use of SNPs in personalized therapy for this disease.
\end{abstract}

Metastatic renal cell carcinoma (RCC) is a heterogeneous disease, and the selection of treatment and the prediction of outcome are currently based mainly on tumor histology. In recent years several drugs have been approved for treatment of advanced RCC, but side effects are limiting their use. If toxic effects could be predicted then better treatment could be provided. Uncovering the genetics that underlies RCC and the pharmacogenetics that controls drug effects is crucial if treatment is to be improved.

The clear cell histological subtype of RCC accounts for more than $75 \%$ of kidney tumors and is presumed to arise from the proximal convoluted tubule of the kidney [1]. Sporadic tumors make up 75 to $85 \%$ of all clear cell RCC, and more than $75 \%$ of such sporadic tumors have been found to have defects in the von Hippel-Lindau ( $V H L)$ gene [1]. The VHL protein is a tumor suppressor and VHL mutations that inactivate suppression lead to transcription of hypoxia-inducible genes, including those

*Correspondence: ganapar@ccf.org

Taussig Cancer Institute, Cleveland Clinic, 9500 Euclid Avenue, Cleveland, $\mathrm{OH} 44195$, USA encoding vascular endothelial growth factor (VEGF), platelet derived growth factor- $\beta$ (PDGF- $\beta$ ), transforming growth factor- $\alpha$ and erythropoietin. The highly vascular characteristic of clear cell RCC and the discovery of a potential central role for VEGF signaling triggered the search for agents that target these pathways for the treatment of clear cell RCC.

Since December 2005, the clinical management of clear cell RCC has been boosted by the approval of several agents that target tumor cells. These include the humanized monocolonal antibody bevacizumab, which targets VEGF, the mammalian target of rapamycin (mTOR) inhibitors temsirolimus and everolimus, and the multitargeted tyrosine kinase inhibitors (TKIs) sorafenib, sunitinib and pazopanib (Box 1) [1]. Despite the clinical efficacy of these agents, which have revolutionized the standard of care, toxicities such as hypertension, myelosuppression (reduction in white blood cells and platelets) and skin reactions such as the palmar plantar dysesthesia that are associated with their chronic use affect the choice of these agents for therapy. The side effects caused by TKI therapy have been attributed to their potency at inhibiting VEGF receptors (VEGFRs) and Flt-3 [2,3].

TKIs provide a promising clinical outcome and so there is a need to manage the accompanying toxicity. Substantial effort has been directed at identifying SNPs that can predict activity and/or toxicity, and a recent publication by Garcia-Donas et al. [4] in the Lancet Oncology is another step in the right direction. The authors [4] provide data demonstrating that a panel of selected SNPs can be useful in predicting the activity or toxicity that develops during sunitinib treatment. This is the first prospective study in previously untreated patients, and it evaluates various outcome measures in patients with metastatic clear cell RCC being treated with sunitinib. The study used a panel of 16 key polymorphisms in 9 genes that are linked to the mechanism of action, metabolism and transport of sunitinib to evaluate SNPs in germline DNA isolated from peripheral blood or saliva.

The prospective nature of this research is important; however, the study [4] was conducted in a practice 


\begin{tabular}{l} 
Box 1 Systemic therapy for locally advanced or \\
metastatic RCC \\
\hline Multikinase inhibitors \\
Sunitinib \\
Pazopanib \\
Sorafenib \\
VEGF inhibition and cytokine \\
Bevacizumab and interferon-a \\
mTOR inhibitors \\
Everolimus \\
Temsirolimus \\
Cytokines \\
IL2 \\
Interferon-a \\
Chemotherapy
\end{tabular}

setting, with no protocol guidance for investigators regarding dose levels, dose adjustments and clinical evaluations. As an example, $10 \%$ of patients received starting doses of less than the recommended standard level of $50 \mathrm{mg} /$ day of sunitinib. The primary determinant of efficacy used in this work is progression-free survival (PFS); however, in an uncontrolled setting determination of PFS is sometimes problematic because of the risk of investigator and/or patient bias. In addition, no data are provided about the frequency of missed scans, which can influence PFS determination [5], and 11 of 101 (>10\%) patients were eliminated from the analysis for various reasons. Therefore, the clinical trial design and data collection procedures are unclear and may represent critical issues for evaluation of the SNP data. Finally, the optimal efficacy endpoint is overall survival; use of surrogates such as PFS and/or response may be acceptable if overall survival is confounded by the study design or subsequent therapy. A limitation is that this study and others have evaluated several overlapping SNPs for response and/or toxicity following treatment with sunitinib in patients with metastatic clear cell RCC, yet there is no consensus on a set of 'predictive' SNPs. Despite these drawbacks, the authors [4] identified polymorphisms in the cytochrome P450 gene CYP3A5*1 and VEGFR3 that correlate with tolerability and response, respectively, to sunitinib treatment.

The strategy to evaluate germline DNA as described in this study and used by others certainly provides a convenient and reliable source of high quality DNA for SNP analysis. Thus, one would expect that, at least with enzymes involved in sunitinib metabolism, such as CYP3A5*1 (rs776746), polymorphisms in the germline DNA should provide consistent data for toxicity between studies [4,6]. The data of Garcia-Donas et al. [4] clearly outline a significant role for allelic genotypic differences in CYP3A5*1 (rs776746) that are correlated with dose reductions, whereas that of van der Veldt et al. [6] describes a significant correlation with PFS for the same polymorphism. Similarly, VEGFR3 (rs307826) had an effect on PFS in the study by Garcia-Donas et al. [4] but a similar association was not reported in the study by van der Veldt et al. [6].

As the study by Garcia-Donas et al. [4] exclusively evaluated untreated patients, whereas the van der Veldt et al. [6] study examined treatment-naïve and previously treated patients, previous treatment may be relevant in defining the role of a specific SNP. Garcia-Donas et al. [4] identified two VEGFR3 polymorphisms (rs307826 and rs307821) that had a significant effect on PFS. However, an obvious piece of data that is lacking in all studies evaluating SNPs in TKI-treated patients [4,6-9] is the effect of dose or of dose modifications on pharmacokinetics and circulating VEGF/VEGFR levels. Also, is there a correlation between genotype frequency for a particular SNP in germline DNA and the paired genomic tumor DNA from the same patient? The study by Kim et al. [9] indicated a greater than $98 \%$ correlation between the genotype for VEGF and VEGFR2 SNPs in paired germline and tumor DNA, suggesting that using germline DNA for analysis of SNPs in patients treated with TKIs could be informative.

Another important aspect is the effect of previous treatment on PFS. For example, $\mathrm{Xu}$ et al. [8] evaluated the efficacy of the TKI pazopanib in treatment-naïve and previously treated patients and identified polymorphisms in the interleukin 8 (IL8), hypoxia-inducible factor 1 alpha $(H I F 1 A)$ and VEGFA genes that were associated with PFS or response rate. Although these data are related to treatment with pazopanib and not sunitinib, this information should be considered in the context of a patient who is refractory to sunitinib being subsequently treated with sorafenib, pazopanib or an mTOR inhibitor. Thus, delineating the predictive role of SNPs in treatment-naïve and previously treated patients could be important in defining SNPs as a biomarker on which to base the choice of drug for therapy.

A further consideration is that because germline DNA is used for analysis of SNPs, the role of the host response to the TKI or mTOR inhibitor becomes paramount, because the precise mechanism of action for anti-tumor activity of these targeted agents is yet to be defined. It would also be useful to identify a subset of SNPs from different genes, for example, those encoding VEGF and VEGFR2, associated with a signaling pathway and outcome, as described by Kim et al. [9] in their study evaluating metastatic clear cell RCC patients treated with sunitinib, because this could emphasize the relative 
significance of certain SNPs based on previous therapy and the targeted therapy of choice.

In summary, the interesting data from Garcia-Donas et al. [4] provide additional information on the association of SNPs with response and toxicity in sunitinib-treated patients. They also raise important considerations for trials with TKI or mTOR inhibitors, and we have four recommendations for future clinical trials. Firstly, pharmacokinetics will need to be defined to better understand the relevance to response and toxicity; secondly, the potential differences in treatment-naïve and pre-treated patients need to be included; thirdly, study design and endpoint evaluation must be prospective and include a well-designed protocol, with sufficient patient numbers, and critical evaluation of toxicity and efficacy endpoints; and finally, recommended guidelines for defining a biomarker [10] need to be considered, so that SNPs truly enter the arena of personalized targeted therapy for clear cell RCC.

\section{Abbreviations}

CYP, cytochrome P450; mTOR, mammalian target of rapamycin; PFS, progression-free survival; RCC, renal cell carcinoma; SNP, single nucleotide polymorphism; TKI, tyrosine kinase inhibitors; VEGF, vascular endothelial growth factor; VEGFR, VEGF receptor; VHL, von Hippel-Lindau.

\section{Competing interests}

RNG has previously received research funding from Bayer, Novartis and Pfizer for studies in renal cell carcinoma. RMB is a consultant and speaker for Pfizer, Genentech, Novartis and GSK, and a consultant for BMS and Argos.

Published: 30 December 2011

\section{References}

1. Escudier B, Albiges L: Vascular endothelial growth factor-targeted therapy for the treatment of renal cell carcinoma. Drugs 2011, 71:1179-1191.

2. Bhargava P: VEGF kinase inhibitors: how do they cause hypertension? Am J Physiol Regul Integr Comp Physiol 2009, 297:R1-R5.
3. Kumar R, Crouthamel MC, Rominger DH, Gontarek RR, Tummino PJ, Levin RA, King AG: Myelosuppression and kinase selectivity of multikinase angiogenesis inhibitors. Br J Cancer 2009, 101:1717-1723.

4. Garcia-Donas J, Esteban E, Leandro-García LJ, Castellano DE, Del Alba AG, Climent MA, Arranz JA, Gallardo E, Puente J, Bellmunt J, Mellado B, Martínez E,Moreno F, Font A, Robledo M, Rodríguez-Antona C: Single nucleotide polymorphism associations with response and toxic effects in patients with advanced renal-cell carcinoma treated with first-line sunitinib: a multicentre, observational prospective study. Lancet Oncol 2011 12:1143-1150.

5. Fleming TR, Rothman MD, Lu HL: Issues in using progression-free survival when evaluating oncology products. J Clin Oncol 2009, 27:2874-2880.

6. van der Veldt AA, Eechoute K, Gelderblom H, Gietema J, Guchelaar HJ, van Erp NP, van den Eertwegh AJ, Haanen JB, Mathijssen RH, Wessels JA: Genetic polymorphisms associated with a prolonged progression-free survival in patientswith metastatic renal cell cancer treated with sunitinib. Clin Cancer Res 2011, 17:620-629.

7. van Erp NP, Eechoute K, van der Veldt AA, Haanen JB, Reyners AK, Mathijssen $\mathrm{RH}$, Boven E, van der Straaten T, Baak-Pablo RF, Wessels JA, Guchelaar HJ, Gelderblom H: Pharmacogenetic pathway analysis for determination of sunitinib-induced toxicity. J Clin Oncol 2009, 27:4406-4412.

8. Xu CF, Bing NX, Ball HA, Rajagopalan D, Sternberg CN, Hutson TE, de Souza P,Xue ZG, McCann L, King KS, Ragone L, Whittaker JC, Spraggs CF, Cardon LR, MooserVE, Pandite LN: Pazopanib efficacy in renal cell carcinoma: evidence for predictive genetic markers in angiogenesis-related and exposurerelated genes. J ClinOncol 2011, 29:2557-2564.

9. Kim JJ, Vaziri SA, Rini BI, Elson P, Garcia JA, Wirka R, Dreicer R, Ganapathi MK, Ganapathi R: Association of VEGF and VEGFR2 single nucleotide polymorphisms with hypertension and clinical outcome in metastatic clear cell renal cellcarcinoma patients treated with sunitinib. Cancer 2011. doi:10.1002/cncr.26491.

10. McShane LM, Altman DG, Sauerbrei W, Taube SE, Gion M, Clark GM; Statistics Subcommittee of the NCI-EORTC Working Group on Cancer Diagnostics: Reporting recommendations for tumor marker prognostic studies (REMARK). J Nat/ Cancer Inst 2005, 97:1180-1184.

doi:10.1186/gm295

Cite this article as: Ganapathi RN, Bukowski RM: Predicting responses to sunitinib using single nucleotide polymorphisms: progress and recommendations for future trials. Genome Medicine 2011, 3:79. 